\title{
Small Intestinal Mucosa
}

National Cancer Institute

\section{Source}

National Cancer Institute. Small Intestinal Mucosa. NCI Thesaurus. Code C33568.

Mucosa arranged into two fundamental structures - villi and crypts of Lieberkuhn. Villi are projections into the lumen of the small intestine, covered with simple columnar epithelium. Crypts of Lieberkuhn are moat-like invaginations of the epithelium around the villi that extend through the lamina propria down to the muscularis mucosae.

Lymphocytes often invade the epithelium or form solitary lymphoid nodules in the lamina propria. Lymph nodules may form long itudinal aggregations of 30-50 nodules in the lamina propria of the ileum. These large aggregations are called Peyer's patches. The muscularis mucosae has two layers and extends into the intestinal villi, where the smooth muscle cells form a long itudinal bundle in the centre of the villi. The small intestinal mucosa secretes gastrin, somatostatin, cholecystokinin and secretin. 